Volume 19, No 3 International Journal of Radiation Research, July 2021

\title{
Dose verification and plan conformity with three different dosimeters for intensity-modulated radiation therapy plans
}

\author{
A. Yassin'1 ${ }^{*}$, K. M. Elshahat ${ }^{2}$, E. M. Attlah 3 , H. Khaled ${ }^{2}$, A. Kany ${ }^{4}$ \\ ${ }^{1}$ Medical Physicist, Radiation Oncology Department, Al-Azhar University Hospital, Cairo, Egypt \\ ${ }^{2}$ Radiation Oncology department, Faculty of Medicine, Al-Azhar University, Caro, Egypt \\ ${ }^{3}$ National Cancer Institute. Caro, Egypt \\ ${ }^{4}$ Radiation Physics, Faculty of Science, Al-Azhar University, Cairo, Egypt
}

\section{- Original article}

\section{*Corresponding authors: \\ Adel A. Yassin, Ph.D., \\ E-mail: \\ adelyassin79@gmail.com}

Revised: April 2020

Accepted: June2020

Int. J. Radiat. Res., July 2021;

19(3): 703-710

DOI: $10.29252 /$ ijrr.19.2.703

\begin{abstract}
Background: The intensity-modulated radiotherapy (IMRT) enables personalized treatment; the complexity of this technique increased the need for patient-specific quality assurance (QA). Objective: Comparing three dosimeters that common for patient-specific QA of IMRT. Material and Method: cases were planned at Eclipse treatment planning system (TPS) to receive radiotherapy at Unique VARIAN linear accelerator LINAC; Patient-specific QA was performed with three independent dosimeters: Gafchromic films EPT2, Electronic Portal Image Device (EPID), and PTW 2D array. The absolute dose was measured and analysis of $2 \mathrm{D}$ gamma index was performed, then compared with the plan calculated in TPS. Results: Analysis of absolute dose measured have highest difference from dose in Gafchromic film $(89.1 \pm 4) \%$ while EPID had a lower range $(96.6 \pm 1.2)$ and 2Darray showed an agreement up to $(99 \pm 1.2)$ for patient specific QA both EBT2 and EPID enable to compare the measured map with TPS calculations, for plan conformity the gafchromic film enable measurement with lower accuracy even with localized brain tumor, the heterogeneity in lung case slightly affect the EPID measurement, this found also with irregular surface of head and neck and increased depth within pelvic case examine. Measurement with 2D array found to be the optimum dosimeter within different conditions. Conclusion: different parameters might affect the accuracy of gafchromic film including film scanning, storing, and calibration curve. EPID has an average deviation appears in beam fluence and 2D array as a 2D ion chamber found to have the most accurate dosimeter, but still time consuming when compared to EPID.
\end{abstract}

Keywords: IMRT, patient-specific QA, 2D array, EBT2 Gafchromic film and EPID.

\section{INTRODUCTION}

The Patient-specific QA required for accuracy in treatment delivery, and required in routine work, especially with advanced techniques of radiotherapy. In 3D conformal radiotherapy the patient specific QA performed through measurement of dose in the iso-center point (1), but for intensity modulated radiotherapy (IMRT) the arrangement of field combined with number of smaller subfields (segments) to modulate the radiation distributed with the 
exact shape of tumor hence prevent overexposure to healthy surrounding tissues (2). Therefor the quality assurance needs to be performed for absolute dose and map analysis comparing to calculated map in TPS. Different dosimeters are commonly used for patient-specific QA including gafchromic films (3), electronic portal image device (EPID) (4) and 2 D array (5) each dosimeter was examined separately to determine its accuracy in different clinical conditions.

The first dosimeter we examined was EBT2 Gafchromic films, as they were common for patient positioning but measuring calibration curve enable absolute and relative dosimetry in Patient-specific QA (6), Gafchromic films can be used for 3D reference dosimetry (7). Electronic portal image dosimetry (EPID) is another dosimeter used for $\mathrm{QA}$ and combined with LINAC machine, also used for patient positioning and pre-treatment patient-specific QA (8) Compared with other dosimeters, QA with EPID is a relatively easier with lower meantime required, it was examined for QA of prostate with IMRT plans (9) and other clinical sites in both normal and hyper fractionation mood (10). Recently suggested methods to perform 3D plan verification with EPID have beam presented (11) with correction factor required (12). The third dosimeter used for patient-specific QA was PTW 2D ion chamber array as an accurate device (13). The independent QA could be performed in map check (14), the correlation between plan complexity and gamma index analysis in patient-specific quality assurance of volumetric modulated arc therapy revealed Strong correlation between beam complexity and the gamma passing rate (15), a critical evaluation of the PTW 2D-array seven29 and OCTAVIUS II phantom for IMRT and VMAT verification (16).

In this study, we aimed to investigate the accuracy of three dosimeters on patient-specific QA, cases were selected to represent different body sites, they were $1^{\text {st }}$ contoured by a radiation oncologist, and then the IMRT plans were created by a medical physicist. The verification plan was created after acceptance of primary plan, a different plan series named as verification and consist of the same number of beams, the same MLCs, with couch, collimator and gantry angle set to be zero, the plan added to the CT of sheets of slap phantom at the standard condition of each dosimetry system. The aim of this work is to examine the accuracy and conformity of three dosimeters when used for patient specific QA. EBT2 gafchromic films, 2D array and EPID with $3 \mathrm{~mm}$ Distance ToAgreement, $3.0 \%$ Dose difference with ref. to maximum dose.

\section{MATERIALS AND METHODS}

\section{I- Dosimeters, treatment machine and planning system}

This work was performed to examine pre-treatment patient-specific Quality assurance of IMRT plans with three different dosimeters within the same parameters of evaluation. Dosimeters used in this work were (Gafchromic Film EBT2), electronic portal image device (EPID), and PTW 2D array. IMRT plans were performed using the Eclipse treatment planning system (TPS). The accepted plan transferred by Aria network then Pre-treatment dose verification was performed in Varian (Unique) Linear accelerator as a patient-specific QA for each dosimeter, the measured plan fluence map transferred to VeriSoft software to be analyzed and compared with the primary plan.

\section{II- Phantom imaging for plan verification $Q A$}

The construction of multipurpose snap phantom enabled the QA with gafchromic films, and a 2D array phantom was constructed. The pretreatment plans were calculated in Eclipse TPS. Two sets of CT images were performed, one for film analysis and the other for the 2Darray at $10 \mathrm{~cm}$ depth, then delivered to TPS (figure 1) (17). For patient specific QA, the verification plan created at the TPS, then delivered to the treatment machine at gantry 0 and a combined dose distribution from all fields was applied to slap phantom CT for pre-treatment irradiation. The resulting map was transferred to VeriSoft software and compared with the same field arrangement from TPS. $3 \mathrm{~mm}$ Distance ToAgreement, $3.0 \%$ Dose difference with ref. to

Int. J. Radiat. Res., Vol. 19 No. 3, July 2021 
maximum dose regarding film and 2D array. Gamma index $(3 \mathrm{~mm} / 3 \%)$ to compare the delivered dose distribution (with each dosimeter) to the TPS calculated dose.

\section{III- Comparison of absolute dose measured versus calculated in TPS}

The difference between each dosimeter and TPS dose calculated from equation 1 :

\%diff $=((($ measured dose $)-($ Calculated dose $)) /$ Calculated dose $) \times 100$

(1) (17)

\section{IV- beam fluence and matched map with different dosimeters patient-specific QA EBT2 gafchromic film}

The primary use of EBT2 gafchromic film the specification of linearity, dose dependence and energy dependence test were performed to get the curve enable the absolute dose measurement and beam fluence map. The calibration curve performed by gradual exposure to irradiated dose, then measurement of optical density (OD) for each absolute dose, this curve was transferred to VeriSoft software and saved as a calibration curve. That will then transfer the measured map into absolute dose and map of fluence for each individual patient (18).

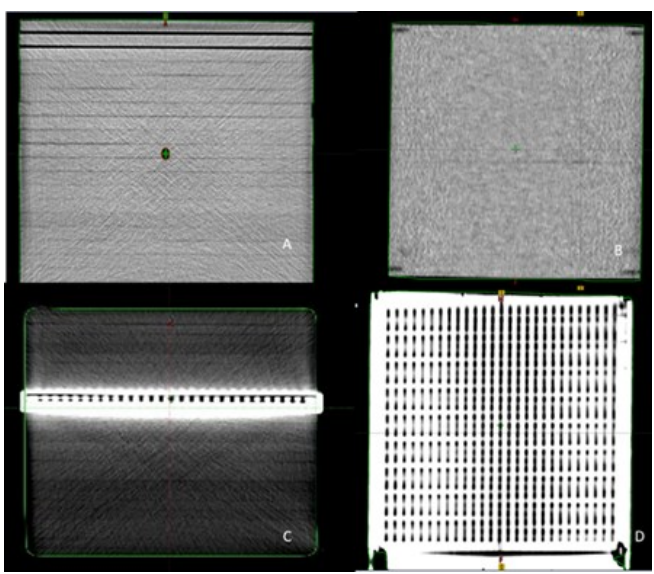

Figure 1. CT-phantom for QA plan in Eclipse: a) axial view of slap phantom, b) frontal view, c) axial 2D array at $10 \mathrm{~cm}$ depth. d) frontal view for 2Darry.

\section{Pre-treatment irradiation}

The dose verification QA performed by complete plan delivery with film horizontal at $10 \mathrm{~cm}$ depth $\left(\mathrm{SSD} 90 \mathrm{~cm}\right.$ ) in $30 \times 30 \mathrm{~cm}^{3}$ of water equivalent sheets, Film irradiation was performed with $100 \mathrm{~cm}$ SSD, film at $10 \mathrm{~cm}$ depth (figure 2)films were irradiated and scanned at Epson 1100 scanner finally the analysis performed at VeriSoft software and compared with corresponding TPS map. The imaging of slap phantom on CT enables it to be used as a multipurpose phantom for patient-specific QA (19).

\section{Patient-specific $Q A$ with $2 D$ array}

The $2^{\text {nd }}$ dosimeter used for patient specific QA was 2D array, it was inserted in $10 \mathrm{~cm}$ depth of slap-phantom, at $100 \mathrm{~cm} \mathrm{SSD}$, and gantry was zero for all fields, the measured map transferred to VeriSoft software for measurement and map fluence analysis (13).

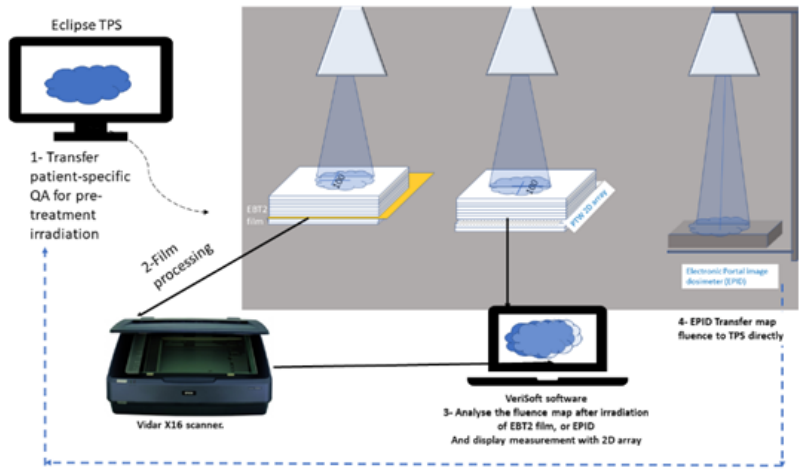

Figure 2. schematic diagram of practical method for $Q A$ in three dosimeters.

\section{Patient-specific QA with EPID}

EPID is an imaging modality attached to LINAC, could be used for pre-treatment dose verification, the receptor is an amorphous silicon, the image acquisition system (IAS) was found to be strongly dependent on the accelerator pulse frequency. This frequency is set for each energy and dose rate, throughout this analysis we kept both dose rate and energy consistence for all plans (20). Pre- treatment irradiation performed at the minimum SSD of $105 \mathrm{~cm}$ with gantry and collimator $\left(0^{\circ}\right)$, the measured plan flounce map transferred via ARIA network to the treatment planning system to be analyzed in Eclipse software $(21,22)$. The portal image resulted map was transferred to TPS for analysis, no additional software required for portal imaging. 


\section{Statistical analysis}

Two-tailed paired t-test was applied to compare the mean of the different measurements of the plans. A p-value of $<0.05$ was significant in the various comparisons.

\section{Ethics committee approval}

The reported measurements performed in this study on the patients were made based on the ethical standards of the institutional and/or national research committee and with the 1964 Helsinki declaration and its later amendments or comparable ethical standards, also our study was performed in slap-phantom as a pre-treatment verification as described in method section.

\section{RESULTS}

The quality assurance for intensity modulated radiotherapy performed in two steps, $1^{\text {st }}$ check the monitor unit deliver the prescribed dose, $2^{\text {nd }}$ check that fields with their segments deliver the dose in pre-defined map to match the planning target volume.

\section{Absolute dose measurement}

Descriptive statistics of absolute dose measured in the central point with the three dosimeters versus the prescribed dose in percentage were illustrated in table 1.

Table 1. Descriptive statics for each dosimeter compared to TPS calculated dose.

\begin{tabular}{|c|c|c|c|}
\hline & EPID & 2D array & gafchromic film \\
\hline Mean & 96.68667 & 99.03333 & 89.12667 \\
\hline Standard Error & 0.301878 & 0.308401 & 1.128314 \\
\hline Median & 96.4 & 99.7 & 90 \\
\hline Mode & 96.2 & 100 & 90 \\
\hline Standard Deviation & 1.169167 & 1.194432 & 4.369941 \\
\hline Range & 3.8 & 2.6 & 18.7 \\
\hline Minimum & 95.2 & 97.4 & 78.3 \\
\hline Maximum & 99 & 100 & 97 \\
\hline $\begin{array}{c}\text { Confidence Level } \\
\text { (95.0\%) }\end{array}$ & 0.647463 & 0.661454 & 2.419992 \\
\hline
\end{tabular}

The absolute dose measured with EBT2 gafchromic film had the highest variance when compared to EBID and 2D array. Percentage of differences between measured and TPS calculated dose shown in figure 3 . Figure 3 Shows Box and whisker blot of absorbed dose through patient-specific QA /TPS calculated dose, the highest difference in film measurements (mainly underestimation up to $-15 \%$ ), while 2D array appears to be the closest (with high range of difference comparing to EPID). This demonstrates the high range of uncertainty through film irradiation.

\section{Analysis of plan conformity in map fluence}

The $2^{\text {nd }}$ test for quality assurance was plan conformity, different body sites were chosen, then patient-specific QA performed with each dosimeters, to examine the effect of different physical parameters including heterogeneity in lung cases nonuniformity in head and neck, also the increased depth within pelvic cases examined in rectum cases, and the level of accuracy within small localized brain cancer.

The accepted dose was verified in three independent dosimeters then compared to planning system calculations as illustrated in figure 4 for brain cancer, Figure 5 for head and neck cancer, figure 6 for lung cancer and figure 7 for rectal case.

\section{Brain case}

Localized brain tumor treated with IMRT, the dose distribution customized to the planning target volume as illustrated in (figure $4 \mathrm{a}$ and $4 \mathrm{~b}$ ). The measurement of three dosimeters illustrated in (figure 4c, d, e and f).

2D ARRY $\square$ EBT2 FILM $\square$ EPID

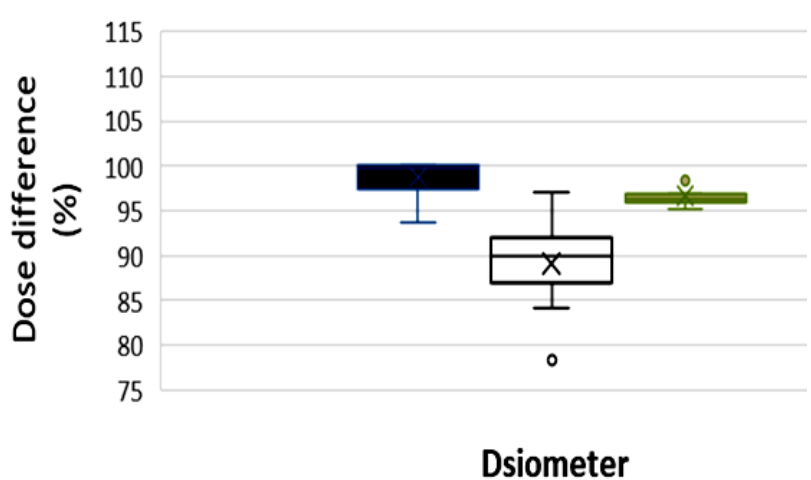

Figure 3. percentage difference with planning calculation.

Int. J. Radiat. Res., Vol. 19 No. 3, July 2021 


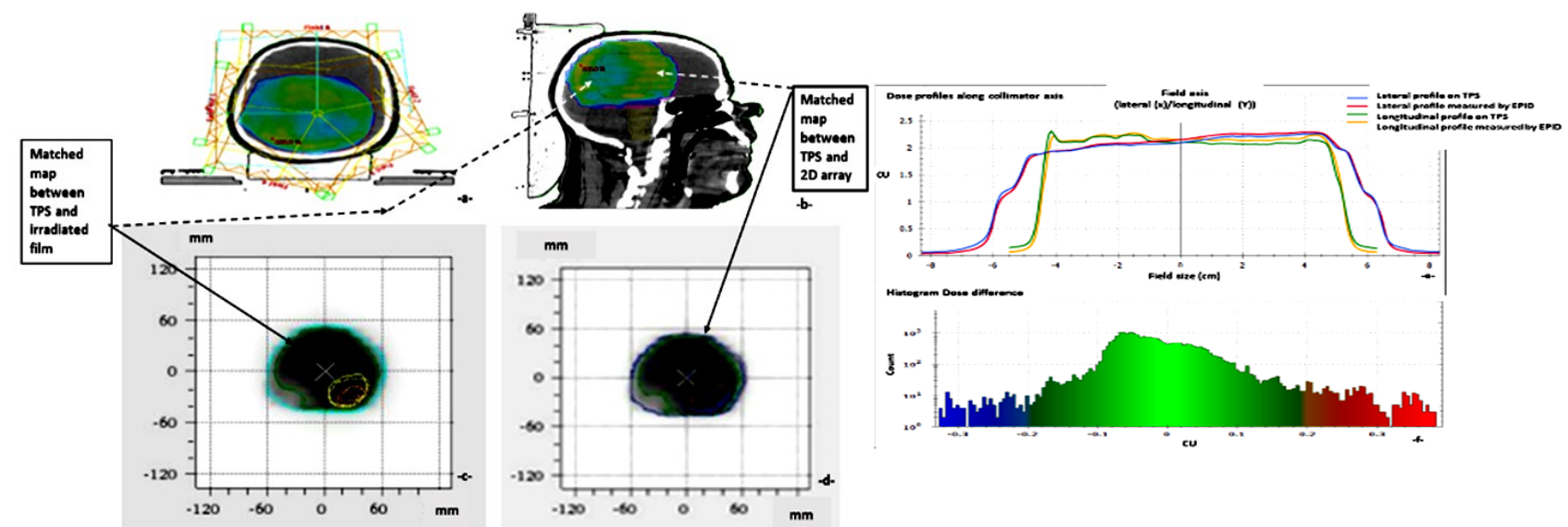

Figure 4. Brain case: a) axial view with beam arrangement, b) Frontal view, c) map measured with EBT2 film, d) 2D array map, e) matched profiles between TPS and EBID. f) histogram dose difference for EBID.

The brain irradiation performed with head fixation, almost no motion available, and the small treated depth help reducing the probability of dose discrepancy. The map measured with EBT2 gafchromic film matched the shape calculated in TPS, with $92 \%$ of absolute dose measurement (figure 4c). The 2D array map (figure $4 \mathrm{~d}$ ) had $100 \%$ accuracy in dose measurement and shows a matched map with TPS as well. The dose measured with EPID represented with beam profile in lateral and longitudinal direction, beside composite of measurements with accumulated fields (figure $4 \mathrm{e}$ and $4 \mathrm{f}$ ).

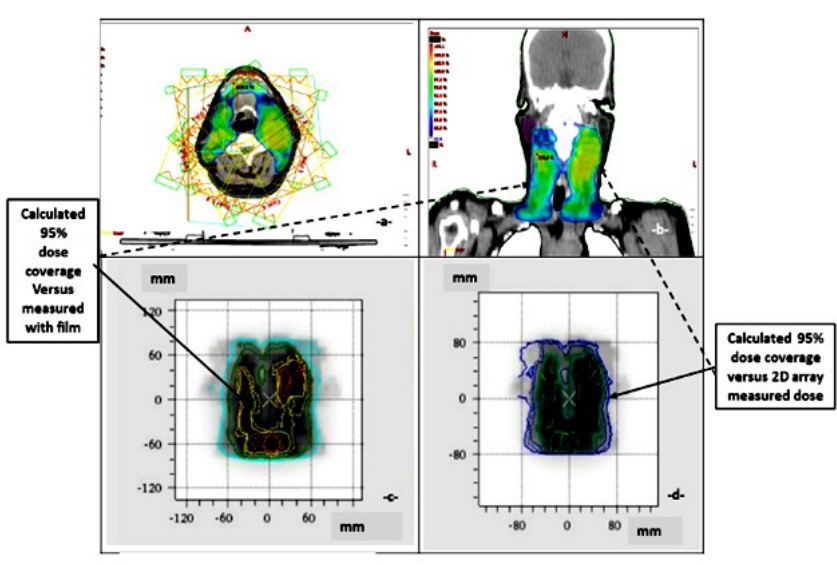

\section{Head and neck case}

Planning nasopharyngeal cases with IMRT enables the patient to receive high dose while sparing spinal cord from exceeding the tolerance. Localized IMRT plan was performed as shown in figure 5 with the measured map for two dosimeters (films and 2Darray) displayed in (figure $5 \mathrm{c}, 5 \mathrm{~d}$ ), also matched plan profiles of EPID measurements displayed in (figure $5 \mathrm{e}, 5 \mathrm{f}$ ).

The head and neck case examined here had a 96\% match with 2D array, 87.9\% match with film map and $96.2 \%$ match with EPID beam profile. The irregularity within head and neck site highly influences the accuracy of the Gafchromic EBT2 film.

Figure 5. Head and neck case: a) axial view with beam arrangement, b) Frontal view, c) map measured with EBT2 film, d) 2D array map, e) matched profiles between TPS and EBID. f) histogram dose difference for EBID. 

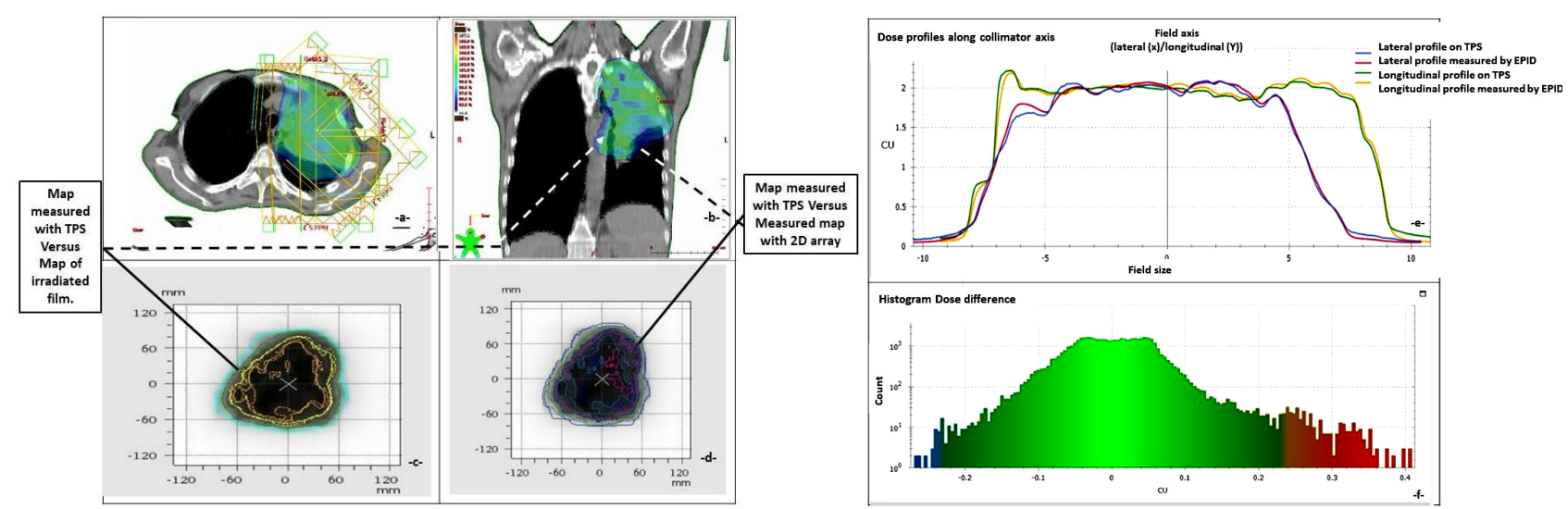

Figure 6. Lung case a) axial view with beam arrangement, b) Frontal view, c) map measured with EBT2 film, d) 2D array map, e) matched profiles between TPS and EBID. f) histogram dose difference for EBID.

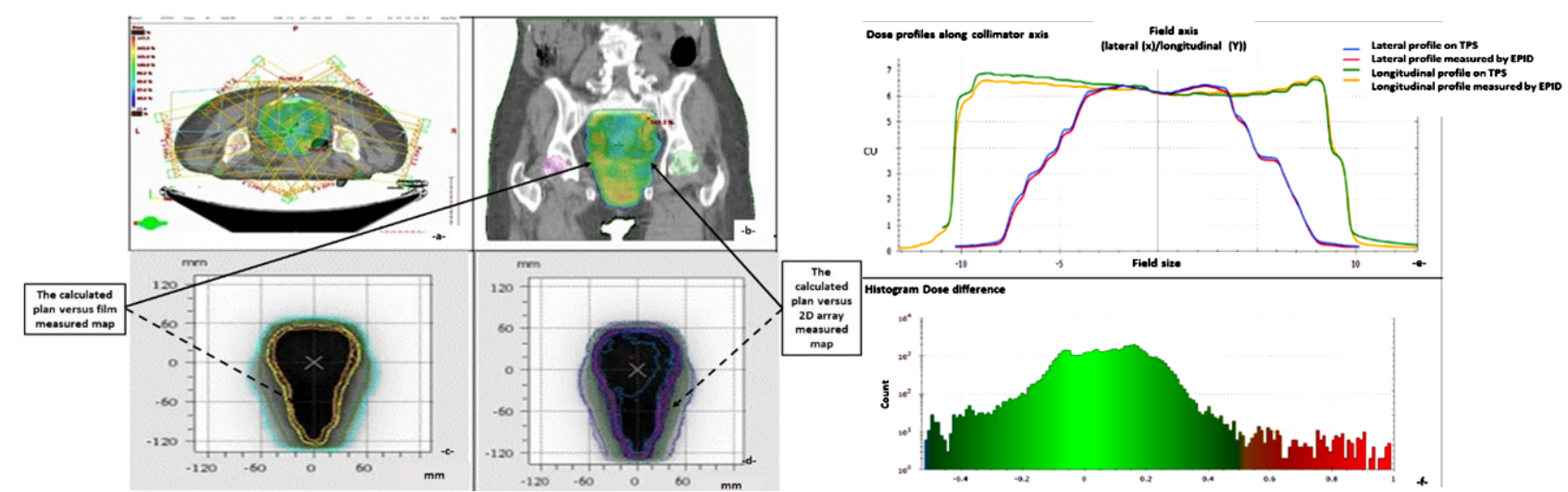

Figure 7. Rectum case: a) axial view with beam arrangement, b) Frontal view, c) map measured with EBT2 film, d) 2D array map, e) matched profiles between TPS and EBID. f) histogram dose difference for EBID. The measured map of EBT2 gafchromic film matched with calculated IMRT plan, with $90.9 \%$ match ( $9.1 \%$ difference with absolute dose). However, the $2 \mathrm{D}$ array map matches $100 \%$, and the EPID profiles were $96 \%$ match the calculated map. Replacing film by EPID was suggested previously for dosimetry of field-by-field verification with closer agreement for isocentre dose measurement, that EPID dosimetry can be used instead of ionization chamber measurements ${ }^{(9)}$.

\section{Lung case}

The calculation of lung dose combined with heterogeneity within the irradiated region. Localized lung cancer planned with IMRT (figure $6 \mathrm{a}, 6 \mathrm{~b})$ number of beams distributed unilateral to prevent the contralateral lung. The measured map for two dosimeters displayed in (figure 6c, 6d) with measured profile of EPID in (figure 6e, $6 f)$.

The represented lung case had a $100 \%$ match when measured with 2D array, versus $88.7 \%$ matches when measured with EBT2 film, and 95\% match with EPID measurements.

\section{Rectum case}

Pelvic cases represent a region of high separation in radiotherapy. The rectum case illustrated in figure 7.

\section{DISCUSSION}

\section{Previous measurements demonstrate the following}

Different parameters need to be considered through patient-specific QA, double check the calculated monitor unit through absolute dose measurement, besides comparing the resulted map, with comparing gamma index analysis also recommended for uniform irradiated region matched with calculated plan in TPS.

The absolute dose measured with 2D array as a 2D ion chamber found to be the most accurate dosimeter with limited variance. EPID had a

Int. J. Radiat. Res., Vol. 19 No. 3, July 2021 
range of uncertainty but within accepted range, and EBT2 gafchromic films had a wider range, and not recommended for absolute dose measurement. Previous studies examined gafchromic film used for double check of irradiated versus light field through machine specific QA, others recommend gafchromic film for absolute dose measurement. Still the type of film may affect the accuracy, in a dosimetric study compared EBT2 with XR-RV3 gafchromic films (18).

The map resulted in EBT2 irradiation found to be a bit pail and affected with irregularity in head and neck cases or heterogeneity within lung cases. EBT2 gafchromic film dosimeter approved to have artifacts that negatively impact the accuracy and precision of film dosimetry measurements (23), even EBT3 in some publications approved to have range of variance (5), beside considering film dosimetry as a time consuming device when compared to other devices $(4,24)$. Those outcomes in our study and previous literatures lead to the need of wider range of acceptance when considering gafchromic film for plan verification.

For patient specific QA, the matched map was the only tool available with gafchromic films. EPID and 2D array had the ability to match the measured dose across lateral and longitudinal direction. Beside an evaluation of each segment (pixel unit) within the irradiated region due to presence of 729 ion champers within the measured region. And number of sensors in the EPID (22).

Electronic portal imaging device (EPID) can be used for daily imaging for treatment localization and verification (19). EPID is an accurate dosimeter and being attached to the linac provide time saving even when involved in routine work. The absolute dose measured with EPID had consistency that was observed across different sites. Previous study suggested EPID to check IMRT MU calculation in an agreement within $3.5 \%$ in $97 \%$ for different treatment sites. However, some difference was observed in some plans. This indicates, in case of high difference the need for another patient-specific QA dosimeter. Hence the cause of these observed large differences may be caused by different physical, biological and dosimetry factors. The underestimation in EPID analysis within $(-3.8 \pm 1.16)$ while Previous publication with $9 \%$ underestimation recommended further investigation, and explain it as (of un known reason) (20) other revealed the underestimation to increased dose/fraction especially with hypo-fractionated IMRT treatments (21). Still EPID considered as an accepted dosimeter for Pretreatment verifications either in all fields or field by field. And the major benefit of EPID is the simplicity performing without need of external dosimeters (22).

The 2D array in this work was the most accurate and approved to have good agreement

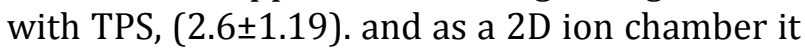
is considered to be an independent tool of dosimetry, being 2D allows accurate measurement of plan fluence, with independent comparative software $(14,15,16,24)$. Also being a Phantom based method for dosimetry audits with independent software enable the plan quality to be checked either in number of cases of the same center or among different centers (25). It was suggested for the routine measuring of the photon beam profiles as alternative to water phantom (26).

\section{CONCLUSION}

Patient-specific QA is mandatory with advanced techniques in radiotherapy, measurement with EBT2 gafchromic film combined with a high range of uncertainty, and not recommended for absolute dose measurement. The accuracy of both EPID found to be accepted, and the 2D array is the only dosimeter that could be used for absolute dose measurement. Each device characterized by certain measurement range, correction factor with calculated uncertainty must be determined pre-clinical application. Even though this work enables us to get the overall range of accuracy within different dosimeters and a number of clinical sites. Further studies with each position still required for results with less variance within each dosimeter. 


\section{ACKNOWLEDGEMENTS}

The authors would like to thank The Children Cancer Hospital (57357) for their cooperation in material supplements.

\section{Financial Disclosure}

The authors declared that this study has received no financial support.

\section{Conflicts of interest: Declared none.}

\section{REFERENCES}

1. Gadhi MA, Azeem N, Azeem A, Fatmi S, Nazir A, Buzdar SA (2019) Quantitative measurements for entrance and exit radiation dose confirmation for cancer patients: An analysis of large cohort of patients. Int J Radiat Res, 17(3): 415420.

2. McKenzie EM, Balter PA, Stingo FC, Jones J, Followill DS, Kry SF (2014) Toward optimizing patient specific IMRT QA techniques in the accurate detection of dosimetrically acceptable and unacceptable patient plans. Med phys, 41 (12): 121702

3. Tanooka M, Doi H, Miura H, et al. (2013) Threedimensional radiochromic film dosimetry for volumetric modulated arc therapy using a spiral water phantom. Int J Radiat Res, 54(6): 1153-1159.

4. Mahdavi SR, Gharehbagh, EJ, Mofid B, Jafari A H, Nikoofar AR (2017) Accuracy of the dose delivery in prostate cancer patients-using an electronic portal imaging device (EPID). Int J Radiat Res, 15(1), 39.

5. Hassn H, Deiab SNA, Aly AH (2020) Dosimetric study of photon beam characteristics with $2 \mathrm{~d}$ array and water phantom measurement. Int J Radiat Res, 18(1): 167-172.

6. Ezzell GA, Galvin JM, Low D, Palta JR, Rosen I, Sharpe MB, Cedric XY (2003) Guidance document on delivery, treatment planning, and clinical implementation of IMRT: report of the IMRT Subcommittee of the AAPM Radiation Therapy Committee. Medical physics, 30(8): 2089-2115.

7. Devic HS (2011) Radiochromic film dosimetry: past, present, and future. Physica medica, 27(3):122-134.

8. Van Esch A, Bohsung J, Sorvari P, et al. (2002) Acceptance tests and quality control (QC) procedures for the clinical implementation of intensity modulated radiotherapy (IMRT) using inverse planning and the sliding window technique: experience from five radiotherapy departments. Radiother and oncol, 65(1): 53-70.

9. McDermott LN, Wendling M, Van Asselen B, et al. (2006). Clinical experience with EPID dosimetry for prostate IMRT pre-treatment dose verification. Med phys, 33(10): 39213930.

10. Van Elmpt W, Nijsten S, Mijnheer B, Dekker A, Lambin P (2008) The next step in patient-specific QA: 3D dose verification of conformal and intensity-modulated RT based on EPID dosimetry and Monte Carlo dose calculations. Radio- therapy and oncology, 86: 86-92.

11. McDermott LN, Wendling M, Nijkamp J, Mans A, Sonke JJ, Mijnheer BJ, van Herk M (2008) 3D in-vivo dose verification of entire hypo-fractionated IMRT treatments using an EPID and cone-beam CT. Radiotherapy and oncology, 86:35-42.

12. Wendling $M$, McDermott $L N$, Mans $A$, Sonke JJ, van Herk M, Mijnheer BJ (2009) A simple backprojection algorithm for 3D in vivo EPID dosimetry of IMRT treatments. Med phys, 36: 3310-3321.

13. Spezi E, Angelini AL, Romani F, Ferri A (2005) Characterization of a $2 \mathrm{D}$ ion chamber array for the verification of radiotherapy treatments. Phys. in Med., Biol., $50: 3361$.

14. Jursinic PA, Sharma R, Reuter J (2010) MapCHECK used for rotational IMRT measurements: step and shoot, TomoTherapy, RapidArc. Med phys, 37: 2837-2846.

15. Stasi M, Bresciani S, Miranti A, Maggio A, Sapino V, Gabriele $P$ (2012) Pretreatment patient specific IMRT quality assurance: a correlation study between gamma index and patient clinical dose volume histogram. Med phys, 39: 7626-7634.

16. Hussein M, Adams EJ, Jordan TJ, Clark CH, Nisbet A (2013) A critical evaluation of the PTW 2D ARRAY seven29 and OCTAVIUS II phantom for IMRT and VMAT verification. Journal of applied clinicalmedical physics, 14: 274-292.

17. Esmaili G, Mahdavi SR, Nikoofar AR, Fadavi P, Ameri A, Nazari V (2018) Dosimetric verification of pre-treatment intensity modulated radiation therapy in the commissioning process. Int J Radiat Res, 16:493-497.

18. Setilo I and du Plessis FCP (2016) Dosimetric comparison between XR-RV3 and EBT2 radiochromic film in megavoltage photon beams. Int J Radiat Res, 14(2): 149.

19. Won HS, Chung JB, Eom KY, Hwang DG, Kang SW, Suh TS (2018) Feasibility study of multi-purpose quality assurance phantom for pretreatment verification of volumetric modulated arc therapy. Int J Radiat Res, 16(3): 279-287.

20. Mahdavi SR, Gharehbagh EJ, Mofid B, Jafari AH, Nikoofar AR (2017) Accuracy of the dose delivery in prostate cancer patients-using an electronic portal imaging device (EPID). Int J Radiat Res, 15(1): 39.

21. McDermott LN, Wendling M, Nijkamp J, et al. (2008) 3D invivo dose verification of entire hypo-fractionated IMRT treatments using an EPID and cone-beam CT. Radiotherapy and oncology, 86(1): 35-42.

22. Grządziel A, Smolińska B, Rutkowski R, Ślosarek K (2007) EPID dosimetry-configuration and pre-treatment IMRT verification. Reports of Practical Oncology \& Radiotherapy, 12(6): 307-312.

23. Lynch BD, Kozelka J, Ranade MK, Li JG, Simon WE, Dempsey JF (2006) Important considerations for radiochromic film dosimetry with flatbed CCD scanners and EBT film. Medical physics, 33: 4551-4556.

24. Nalbant N, Kesen D, Hatice B (2014) Pre-treatment dose verification of IMRT using gafchromic EBT3 film and 2D array. J Nucl Med Radiat Ther, 5(2).

25. Pasler M, Hernandez V, Jornet N Clark, CH (2018) Novel methodologies for dosimetry audits: Adapting to advanced radiotherapy techniques. Physics and Imaging in Radiation Oncology 5: 76-84.

26. Hassn S, Deiab NA, Aly AH (2020) Dosimetric study of photon beam characteristics with $2 \mathrm{~d}$ array and water phantom measurement. Int J Radiat Res, 18(1): 167-172.

Int. J. Radiat. Res., Vol. 19 No. 3, July 2021 